\title{
Low-cost microcomputer networking: How to get high throughput on a low budget
}

\author{
ADAM V. REED \\ Graduate Faculty of Social and Political Science \\ New School for Social Research, New York, New York 10011 \\ DANIEL LEWART \\ Holmdel High School, Holmdel, New Jersey 07733 \\ and \\ LINDA H. SCHNEIDER \\ New York University, New York, New York 10003
}

\begin{abstract}
A very low-cost, high-throughput laboratory data acquisition and experiment control system may be configured by using a star network architecture with a low-cost microcomputer as network controller and one or more microprocessor-based single-board controllers as satellites. A network of this type, using Apple II microcomputer 181,600 with 48K RAM and one minidiskette drive) as main node and up to seven KIM-1 microcontrollers (8169 each) as satellites is described, and its development is discussed in detail.
\end{abstract}

This paper describes the local network approach to the problem of increasing the throughput of laboratory computer systems. The main advantage of this approach is low cost. A rough rule of thumb used by system planners is that with the traditional single-processor approach, every doubling of throughput triples the cost of the system. With the network approach, each satellite processor adds about $10 \%$ to the cost of hardware for the system.

With this kind of cost advantage, it is rather surprising that the network approach is not more widely used in experimental laboratories. One likely explanation for this is the mystique acquired by networking in the early days of computing. According to this mystique, network development requires sophisticated development systems and large amounts of professional effort. One of our goals for this paper is to show how outdated this mystique has become in the last couple of years.

The microcomputer network described in the present paper was developed with the network controller, a standard integer-version Apple II microcomputer, as its own resident development system. The software development effort took a total of $10 \mathrm{~h}$ of the first author's time, as well as about $16 \mathrm{~h}$ of spare time contributed by the second author while he was a student at Holmdel High School. The hardware interface was built by the third author in slightly under $5 \mathrm{~h}$. While a psychologist who is not also a computer engineer may have to add between $\$ 500$ and $\$ 1,000$ in consultants' fees to the cost of the network described in this paper, a microcom-

Daniel Lewart is now at the Massachusetts Institute of Technology. puter network could still constitute the most costeffective approach to adequate system throughput.

A computer network is a system of two or more independently programmable processors, at least one of which is capable of exerting complete control over all the others under the direction of its own program. Our design objectives were to provide adequate throughput for current experiments, to provide future expansion capability up to twice the maximum requirements of any single foreseeable experiment in cognitive psychology, and to minimize development time for the present system as well as for future experiments. Note that a capability for simultaneous execution of several experiments was not one of our design objectives. In general, the most cost-effective way of running several experiments is to run them on separate systems.

\section{HARDWARE}

The Central (Controller) Node

The Apple II was originally chosen as a laboratory computer primarily because its all-socketed construction facilitated the hardware modifications (Reed, 1979) necessary for tachistoscopic stimulus presentation and accurate timing of stimulus-response latencies. Socketed construction also minimizes down time, since most malfunctions may be repaired on site by removing the malfunctioning integrated circuit (IC) from its socket and plugging in a replacement. The Apple also meets both requirements for a central node in a local computer network. The first of these requirements is that the central node be equipped with digital input and output lines capable of communicating with satellite processors. 
The other requirement is that the central node be usable as a development system for real-time routines being developed for implementation on the satellite processor. If real-time program development is being done in assembly language, this means that central node software must include a symbolic assembler capable of assembling code to an object (OBJ) location in random-access memory (RAM) other than the actual logical origin (ORG) the program is to have on the system on which it will be eventually executed. In general, any assembler having separate $\mathrm{OBJ}$ and ORG pseudooperations or assembler directives will be capable of doing the job. The assembler included in the Microproducts, Inc., development system package for the Apple II is equipped with these features, and it is this package that is currently used on the network to develop real-time software for the KIM-1. Unfortunately, assemblers seldom have the above features in the same package with interactive assembly capabilities necessary for network development.

The networking software for the Apple II was actually developed without a symbolic assembler. We used the interactive miniassembler included in the firmware of the standard integer-version Apple II. The absence of symbolic labeling capability in this firmware was more than made up for by its interactive debugging and structuring features. The integer-version Apple II is equipped with a debugging monitor that has go (run), list (disassemble), trace, single step, register examine, and register fill commands, as well as multiple command sequence capability. Any debugging monitor command sequence may be executed without leaving the miniassembler. Moreover, the go command emulates a JSR subroutine call, a feature that encourages FORTH-like structured programming. An interactive assembler of this type facilitates the segmenting of code into short externally callable subroutines, each of which is fully debugged and tested at the time it is written, and before the programmer goes on to write the next segment.

\section{The Satellite Node(s)}

The microcomputer/microcontroller selected for the function of a satellite in a laboratory microcomputer network must satisfy four criteria. First, the instruction set of its processor should be the same as that of the central node's, a condition essential to uncomplicated cross-development of real-time software for experiments. Second, an external device, capable of being simulated by the central node processor, should be able to load desired information into the satellite's memory, to obtain a dump of the satellite's memory on demand, and to start program execution at any desired location in the satellite's memory. Our requirement of reducing development effort to the minimum added an extension of this condition: The software for all of the above functions had to be already present as firmware in the satellite's read-only memory (ROM). This would free us from having to write any real-time networking software for any microcomputer other than the central node, on which adequate development software was known to be available. The third and fourth conditions were the obvious ones: The satellite had to be able to perform the functions necessary to carry out its role in psychological experiments, and it had to fit our budget.

The first of the above conditions narrowed considera. tion to microcomputers using the 6502 processor used in the Apple II. The second condition narrowed consideration to the several industrial microcomputer/ microcontroller boards designed for use with ASR serial terminals, and for using the terminal's local storage facility (originally, the paper-tape facility of the ASR 33 Teletype) for memory loading and dumping and the keyboard of the terminal for control commands, including the command to begin execution at a specified location in memory. The boards in this category included the JOLT and the SUPERJOLT, the KIM-1, the SYM, the SUPERKIM, and the AIM-65. We selected the KIM-1, at $\$ 159$ the second cheapest of the above boards, primarily because the second author was already thoroughly familiar with this unit, having used it as the central component of his personal computer system. With 30 digital input/output (I/O) lines and two precision timers, it clearly had the capability for taking over the task of monitoring and recording subject responses while the Apple dealt with precise presentation of experimental stimuli.

\section{The Hardware Interface}

While the serial interface of the KIM-1 is designed for $20-\mathrm{mA}$ current-loop operation, serial information is actually sent and received for processing at standard 5-V transistor-to-transist or logic (TTL) levels. Hence, we decided to attach the interface cable to the KIM with clip-on connectors bypassing the 20-mA current-loop circuits. The resulting interface is shown in Figure 1. In keeping with our decision to minimize design time, we did not bother to balance the transmission line. The interface shown in Figure 1 works fine to $3 \mathrm{kbaud}$. KIM-1 firmware limits transmission rates to 9.6 kbaud in any case, and the relative improvement obtainable by designing a balanced-line interface would have been limited to that rate. Apart from connectors, the interface shown in Figure 1 consists of three wires and one resistor.

On the Apple II side of the interface, a single KIM-1 is attached to Input SWO and to Output ANO. Three KIM-1s could be connected by using SWO-SW2 and ANO-AN2. A multiplexer routing SWO and ANO according to AN1-AN3 may be used with existing software to run a network comprising up to seven KIM-1s, with one of the eight possible multiplexer codes reserved for loading and starting all the satellites simultaneously.

\section{SOFTWARE}

\section{Teletype Emulation}

In developing network control software for the Apple Il, we minimized development work by making maximum use of published software. Sender software for 


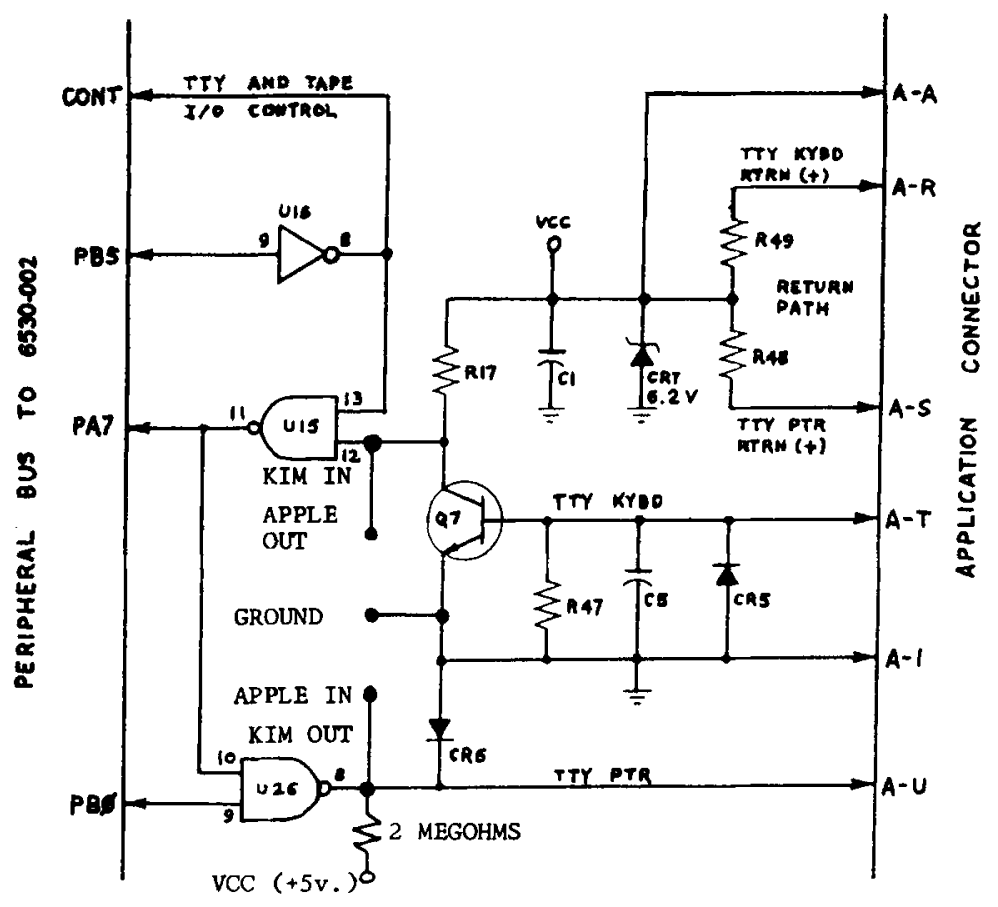

Figure 1. Circuit diagram of the KIM-1 TTY interface circuit and the Apple II/ KIM-I network interface connection.

Teletype emulation was adapted from the TTYDRIVER program (see Apple Computer, Inc., Note 1, p. 119) by $\mathrm{R}$. Wigginton and $\mathrm{S}$. Wozniak. This program was revised to run at 30 times its original baud rate. The KIM-1 TTY input routine was adapted to the hardware available on the Apple II by replacing its timing subroutines by corresponding subroutines from TTYDRIVER (Apple Computer, Inc., Note 1), again modified to run at 3 kbaud.

\section{Network Control}

A $1 \mathrm{~K}$ area of the Apple's memory was set aside for an input map of the KIM's memory. We then wrote a program for the Apple that uses the KIM's built-in TTY dump routine to transfer an exact map of all of KIM's RAM (except for the stack area in the top half of Page 1) to the map area in the Apple. A similar program transfers the content of a similar 1K-byte "output map" area from the Apple to the KIM (taking care not to overwrite RAM locations used by the KIM TTY load routines the program invokes). A third Apple program starts the KIM at a location in KIM-1 RAM specified by 4 bytes of RAM in the Apple II.

A fourth program for the Apple sends out a synchronization pulse that can be read by the sequence

$$
\begin{aligned}
& \text { BIT } 1740 \\
& \text { BPL \#FB }
\end{aligned}
$$

when this sequence is executed by the KIM-1. This simple protocol synchronizes the satellite node(s) to within 6 microsec with the central node and with each other. Finally, we insure the transfer of control back to the Apple by having the Apple wait for its input to go high. The KIM is programmed to return to "TTY" control by ending its program with an unconditional JMP 1 DBC.

Since all Apple II programs are also usable as subroutines, it was a simple matter to combine the above programs into two programs used in actual applications of the network. The first of these maps from the Apple to the KIM and then starts the latter at the desired location. The second waits until the KIM returns to Apple control and then maps the KIM to the Apple. Each of these programs takes approximately $15 \mathrm{sec}$. This is not an unreasonable amount of time, especially since the KIM has an unbroken block of 512 locations usable for data storage. This is enough to store 4 bytes of data from each of 128 trials, a reasonable size for a session in most psychological experiments. If the amount of storage or $\mathrm{I} / \mathrm{O}$ in the $\mathrm{KIM}$ proves inadequate, it may be readily expanded, or additional nodes may be added to the network, and so on.

\section{REFERENCE NOTE}

1. Apple Computer Inc. Apple II Reference Monual, Apple Part No. 030-0004-00. Cupertino, Calif: Author. (Available from Computer, Inc., 10260 Bandley Drive, Cupertino, California 95014).

\section{REFERENCE}

ReEd, A. V. Microcomputer display timing: Problems and solutions. Behavior Research Methods \& Instrumentation, 1979, 11, 572-576. 
Appendix A

Network Software Memory Map (Apple II)

Map of KIM for next invocation of
8200-95FF or 8C40

Appendix B

Network Software Listing

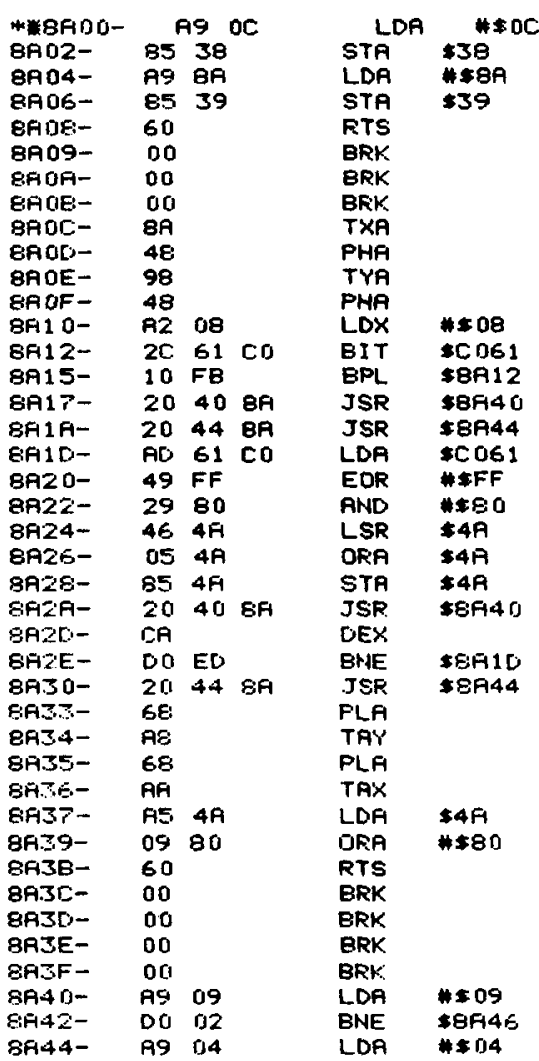

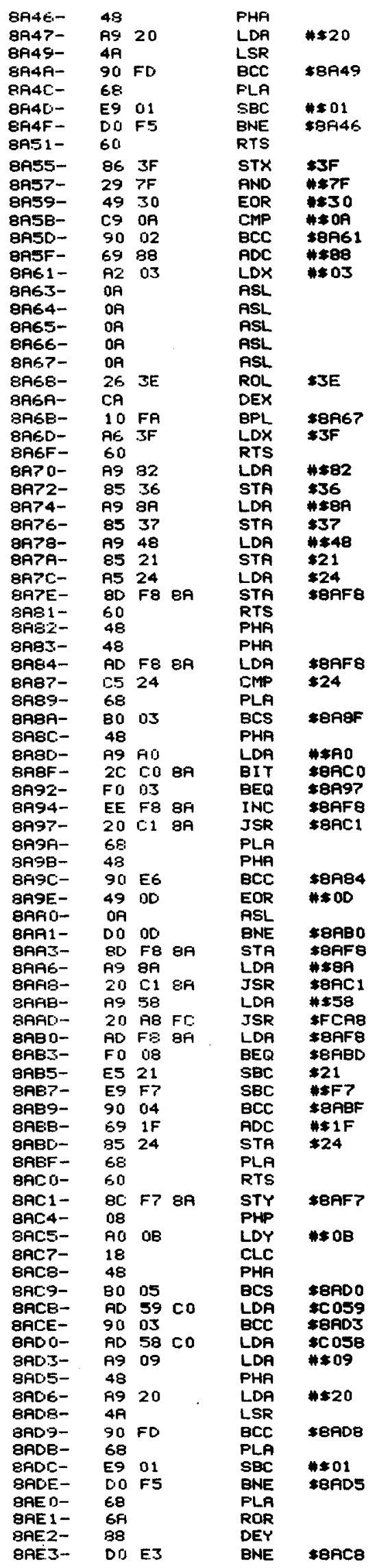




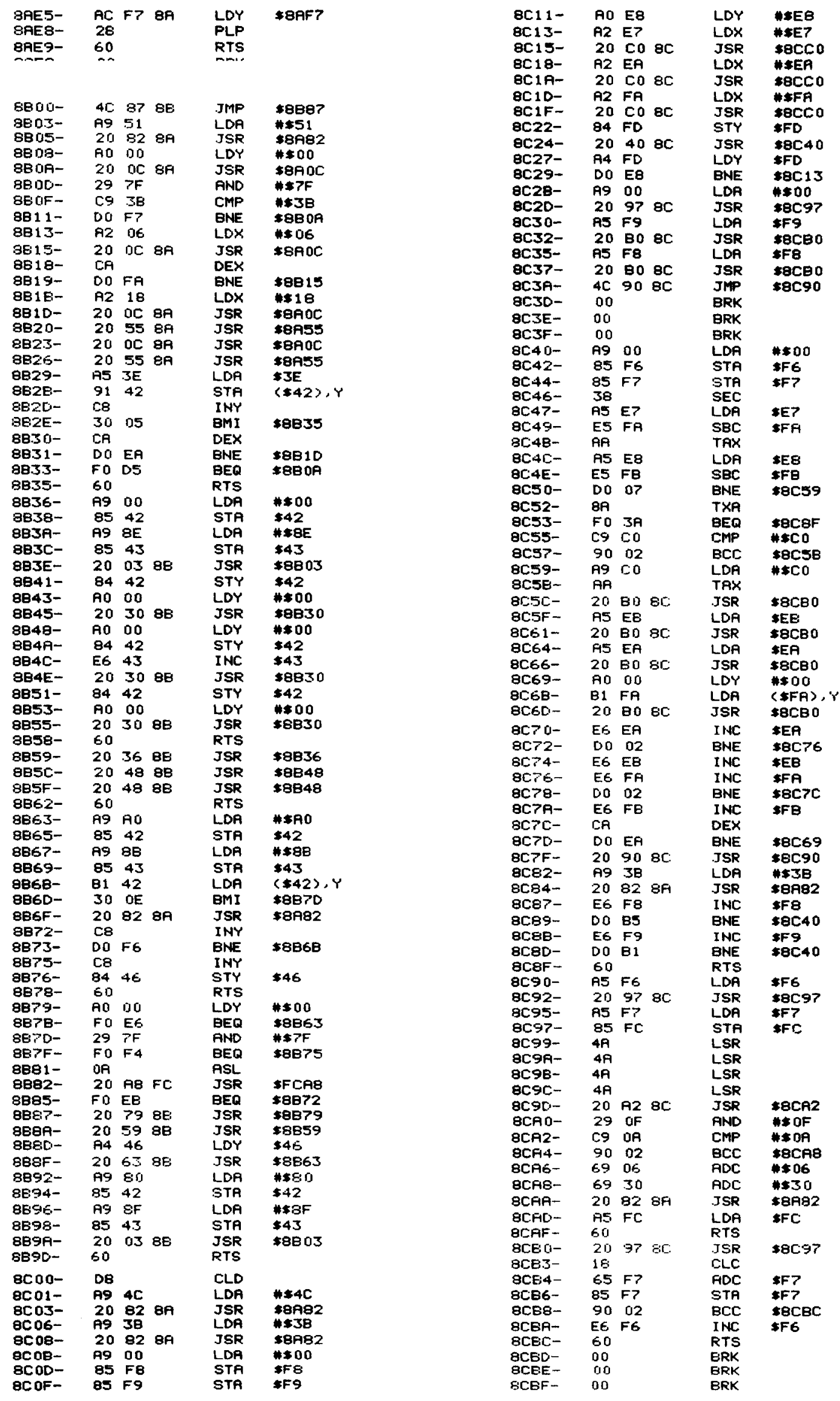




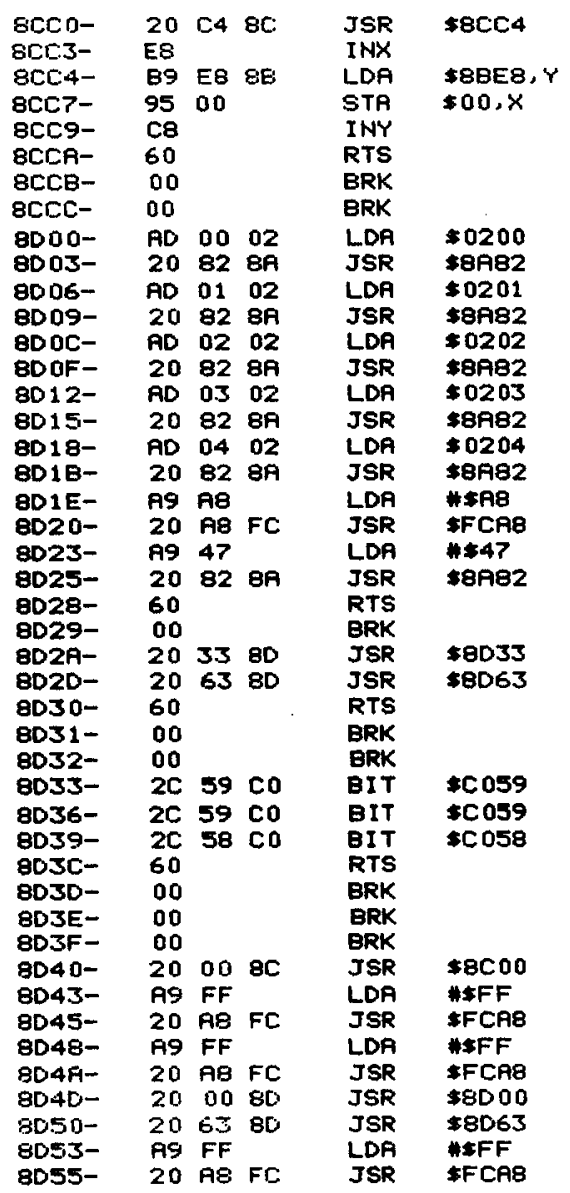

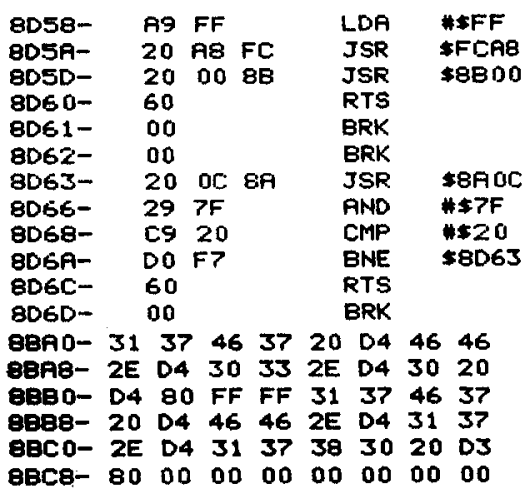

Appendix C

Network Initialization Procedure for Satellites

1. Set KIM-1 KBD-TTY switch to KBD.

2. Press the following sequence of keys on the KIM keyboard:

$$
\text { (RS) (AD) 17F2 (DA) } 18 \text { (+) } 00 \text { (AD) }
$$

This sequence sets the baud rate to 3,000 baud.

3. Set the KBD-TTY switch to TTY.

4. Run appropriate network sof tware from the Apple II. 